Method 2dSearch is a radical alternative to conventional lineby-line query builders. Instead of entering Boolean strings into one-dimensional search boxes, queries are formulated by manipulating objects on a two-dimensional canvas. Query suggestions are provided via an NLP services API, and support is offered for optimising and translating search strategies for different databases. Moreover, strategies can be saved, shared and reviewed as executable artefacts. This approach eliminates many sources of error, makes the query semantics more transparent, and offers an open-access platform for sharing reproducible search templates and best practices.

Results 2dSearch currently supports over 300 registered users (and many more unregistered) in providing an open-access, integrated development environment specifically optimised for search strategy development. It includes:

- A visual framework which eliminates many errors associated with traditional command-line query formulation tools;

- Search results that update in real-time, and individual blocks with hit counts that can be enabled/disabled on demand;

- Queries that are analysed and validated, with common errors (e.g. duplication, orphaned lines, redundant bracketing) detected and corrections offered;

- Platform-agnostic representation and support for multiple databases which mitigates inefficient 'translation' of search strategies across databases;

- Interactive query suggestions that avoid the problems of phrase boundary detection and 'query drift' that undermine traditional query expansion techniques;

- Outputs that may be shared as executable artefacts or exported as traditional Boolean strings.

Conclusions Complying with seven recommended strategies to enhance the reproducibility in scientific research, this platform supports pre-registration of searches, open search strategies (methods), open search results (data), collaboration in review team in search step, automation of validation and translation of search syntax, compatibility with reporting guidelines, and finally pre- and post-publication peer-review.

\section{THE FACT THAT 'THE SCANDAL OF POOR MEDICAL RESEARCH' BY DOUGLAS ALTMAN (1994) LARGELY HOLDS TRUE TODAY INSINUATES THE PERSISTENT CONDUCT AND DISSEMINATION OF POOR-QUALITY RESEARCH. HOWEVER, FUTURE DIRECTIONS PRESENTED IN THE EBM MANIFESTO PROVIDE SOME ASSURANCE THAT RESEARCH QUALITY IN EVIDENCE-BASED MEDICINE CAN BE SALVAGED}

Dhakshenya Dhinagaran. LKC medicine Nanyang Technological University, Singapore, Singapore

10.1136/bmjebm-2019-EBMLive.72

The objective of this submission is to describe four additional suggestions to alleviate issues around the quality of research and publications. Currently, positive results are twice as likely to be published as negative results. Advocating the publication of negative findings too would beseech individuals to report true findings and be less tempted to fabricate data. Moreover, negative findings help expedite the process of uncovering positive results and is supported by 'Missing pieces' by PLOS ONE and the journal 'Negative results'. However, their negligible impact factor and low profile may dissuade researchers from exhibiting their work here. It can be challenging for new journals to compete with gold standards like Nature, NEJM or the BMJ. To tackle this, introducing a subset journal under well-known 'brands' eg. 'Nature/BMJ Negatives' could help attract contributions from researchers. Additionally, the regular updating of reviews calls for more living documents. Universities could collate the appropriate manpower and educational bodies, the necessary funding, to develop field specific documents under open access peer review journals such as the 'Living Journal of Computational Molecular Science.' Living documents help prevent duplication of research, allow for consistent presentation of data and the chronological depiction of findings allows comparisons to be made and trends to be detected as the topic evolves. Many healthcare professionals (HCPs) are not formally trained to conduct research resulting in them addressing questions with low statistical significance or engaging in ghost authorship to climb the career ladder. As such, there is a need to raise the requirements for HCPs who wish to do research (by completing mandatory research training courses) or ensure they are adequately supervised (eg. through collaboration with a career researcher). The feasibility of this suggestion is reiterated by the advent of digital technology which allows HCP's education to seamlessly integrate into their hectic schedules as e-resources can be accessed remotely and at their own convenience. Finally, a reduction in research output is necessary to alleviate the '75 trials and 11 systematic reviews a day' predicament. A revamp of the 'quantity over quality' mind-set is necessary. Researchers should be encouraged to broaden their scope of work beyond publication numbers and impact factors towards conference presentations, teaching, mentoring, contributing to scientific communications etc. These various components contribute towards a rewarding career, unlike a chase for an admirable h-index. To conclude, this proposal targets issues listed in the EBM manifesto. Promoting transparency in research helps reduce questionable research practices. Initiating more living documents elevates the worth of existing evidence through systematic representation of a topic. Furthering HCP education enables them to expand their roles in research. And finally, encouraging a reduction in research output results in evidence being more 'relevant, replicable, and accessible to end users.'

\section{SCIENCE REBOOT. CREATION OF AN EVIDENCE-BASED LITERATURE BY INCREASING THE USE OF REGIONAL LANGUAGES IN SCIENCE}

\footnotetext{
${ }^{1}$ Svjatoslavs Kistkins, ${ }^{2}$ Maksims Mastalers, ${ }^{2}$ Aleksandra Podhvatilina, ${ }^{3}$ Emil Syundyukov, ${ }^{2} J a n a$ Visnevska. 'Latvian Biomedical Research and Study Centre, Riga, Latvia; ${ }^{2}$ University of Latvia, Riga, Latvia; ${ }^{3}$ Longenesis Ltd (Hong Kong Science Park), Hong Kong, China
}

\subsection{6/bmjebm-2019-EBMLive.73}

Objectives English as an International Language is widely used in science. Unfortunately, scientific information space is oversaturated by using International Language, while Regional Languages remain informatively poor. The oversaturation leads to the information overload ${ }^{1-4}$, reducing the quality of information and data interpretation. At the same time, non-English speaking countries publish new studies in regional journals, causing contrary effect - information deficiency in English. ${ }^{5}$

Similarly to RCSB PDB and NCBI, the authors suggest to create a novel scientific database in order to: - Enrich the regional languages allowing them to be competitive; - Concentrate on the evidence-based and up-to-date information in 